\title{
Vortices in Holomorphic Line Bundles over Closed Kähler Manifolds
}

\author{
Steven B. Bradlow* \\ Department of Mathematics, Stanford University, Stanford CA 94305, USA
}

Received August 29, 1989

\begin{abstract}
We apply a modified Yang-Mills-Higgs functional to unitary bundles over closed Kähler manifolds and study the equations which govern the global minima. The solutions represent vortices in holomorphic bundles and are direct analogs of the vortices over $\mathbf{R}^{2}$. We obtain a complete description of the moduli space of these new vortices where the bundle is of rank one. The description is in terms of a class of divisors in the base manifold. There is also a dependence on a real valued parameter which can be attributed to the compactness of the base manifold.
\end{abstract}

\section{Introduction}

Many interesting equations in gauge theory arise as minimizing conditions for gauge invariant functionals. The self- and anti-self dual Yang-Mills equations, the Hermitian-Einstein equation, the Bogomoln'yi monopole equations and the vortex equations are all equations of this sort (cf. $[\mathrm{A}-\mathrm{H}-\mathrm{S}],[\mathrm{F}-\mathrm{U}],[\mathrm{J}-\mathrm{T}])$. Much of the interest in such equations lies in the conditions for existence of solutions and in the moduli space of gauge equivalence classes of solutions (cf. [A-H-D-M], [D1], [D2], [Hi], [H-M], [T]). In this paper we describe a new addition to this collection of "minimizing equations" and address the question concerning the moduli space of its solutions.

In the case of the self- and anti-self dual equations, the functional being minimized is the Yang-Mills functional over $\mathbf{R}^{4}$. The solutions to these equations form a special class of connections on principal bundles over $\mathbf{R}^{4}$. All such solutions have associated to them an integer valued "quantum number" known as the instanton number. Both the existence of the special equations for the extrema and the explanation of the instanton numbers can be attributed to the same thing; the key point is that in four dimensions the Yang-Mills functional can be split as a

\footnotetext{
* Current address: Department of Mathematics, University of California at San Diego, La Jolla CA 92093, USA
} 
sum of a non-negative term and a term which is essentially topological (it is in fact related to the topology of a bundle over $S^{4}$ ). The vanishing of the non-negative term leads to the equations for the minima and the instanton number is related to the topological term. The mechanism used to achieve this is the splitting, via the Hodge star operator, of 2 -forms on $\mathbf{R}^{4}$ into its self-dual and anti-self-dual components.

A similar splitting of the Yang-Mills functional is possible if one replaces $\mathbf{R}^{4}$ by a closed Kähler manifold and considers unitary connections on a complex bundle. In this case however it is the Kähler geometry which enables one to rewrite the functional. The topological part of the functional is clearly seen to be determined by the first and second Chern classes of the bundle and the equations for the minima are now the Hermitian-Einstein equations. In this setting these equations can be interpreted as a constraint on the curvature of a metric connection on a holomorphic bundle. It is an important theorem of Uhlenbeck and Yau [U-Y] that the conditions for the existence of solutions to these equations can be related to the stability (in the sense of Mumford) of the holomorphic bundle.

Taking a slightly different approach, one can view the Yang-Mills functional as a special case of the more general Yang-Mills-Higgs functional. Such a functional is defined whenever one has a vector bundle with compact Lie group as structure group over $\mathbf{R}^{d}$. If $E$ is a vector bundle with structure group $G$ over $\mathbf{R}^{d}, \mathscr{A}$ the space of $G$-connections on $E$, and $\Omega^{0}\left(\mathbf{R}^{d}, E\right)$ the space of sections of $E$, then the Yang-Mills-Higgs functional

$$
\mathrm{YMH}: \mathscr{A} \times \Omega^{0}\left(\mathbf{R}^{d}, E\right) \rightarrow R
$$

is defined by

$$
\mathrm{YMH}(D, \phi)=\left\|F_{D}\right\|_{L^{2}}^{2}+\|D \phi\|_{L^{2}}^{2}+\frac{\lambda}{4} \int_{R^{d}}\left(|\phi|^{2}-1\right)^{2} d \text { vol. }
$$

Here $\lambda \geqq 0$ is a constant, $F_{D}$ is the curvature of $D$ and $D \phi$ is the covariant derivative $[\mathrm{J}-\mathrm{T}]$. Of course all vector bundles over $R^{d}$ are trivializable. One can thus treat the variables as connection 1 -forms and functions on $\mathbf{R}^{d}$. The 1 -forms take values in the Lie algebra of the bundle structure group and the functions take their values in a vector space on which the structure group acts as a transformation group. This is the description frequently used in physics. The connection 1 -form is known as a gauge potential, the vector valued function is called a Higgs field and the vector space in which it takes its values represents a space of internal symmetries for some physical particle (cf. [P], [B1]).

There are certain special cases, other than that of the pure Yang-Mills functional over $\mathbf{R}^{4}$, in which the absolute minima can be described separately from the other critical points. When $d=3$ and $\lambda=0$ one obtains the Bogomoln'yi monopole equations. In dimension two the case where $\lambda=1$ and the vector bundle is a complex line bundle is the special case. This is the so-called Abelian Yang-MillsHiggs model and the corresponding functional is the Abelian Yang-Mills-Higgs functional. Historically, a functional of this type appeared in the theory of superconductivity $[\mathrm{G}-\mathrm{L}]$. There $D$ is taken to represent an electromagnetic potential and $\phi$ to represent a quantum mechanical wave function of an ensemble of Cooper pairs of electrons. $\mathrm{YMH}(D, \phi)$ measures the thermodynamic free energy and the physical configurations are those which minimize the functional.

The global minima of the abelian Yang-Mills-Higgs functional are described 
by two coupled first order equations for the pair $(D, \phi)$. The first equation says that $\phi$ is holomorphic with respect to the holomorphic structure on the line bundle. The second equation is called the vortex equation and its solutions are called vortices. Existence of such vortices was demonstrated by Taubes [T]. The basic result is that solutions always exist and are characterized by the zero set of the section $\phi$ and an integer called the vortex number. The vortex number does have a topological origin, however since the manifold on which the functional is defined is $\mathbf{R}^{2}$, this fact is somewhat obscured.

In the description of these vortices, the base is thought of as $\mathbb{C}$ rather than as $\mathbf{R}^{2}$. It is therefore reasonable to ask whether one could replace $\mathbf{R}^{2}$ by a closed Kähler manifold and then utilize the Kähler structure as was done in the case of the pure Yang-Mills functional over $\mathbf{R}^{4}$.

In this paper we investigate a generalized Yang-Mills-Higgs functional of this sort. In fact we go one step further and modify the Abelian Yang-Mills-Higgs functional so that it is defined for holomorphic vector bundles of any rank over closed Kähler manifolds of arbitrary dimension. The resulting functional still has connections $D$ and sections $\phi$ as its arguments. More importantly, it retains the feature that it is bounded below by topological invariants of the bundle (specifically, a combination of the first Chern class and the second Chern character). One noteworthy difference between our new functional and the one for vortices over $\mathbf{R}^{2}$ is the presence of an extra real parameter $\tau$. The need for this parameter can be attributed to the compactness of the base manifold and the fact that it has finite volume.

It is not hard to identify the conditions for a pair $(D, \phi)$ to minimize our functional. These consist of an integrability condition on the connection, a holomorphicity constraint on the section and an equation which is a direct generalization of the vortex equation. We have attempted to answer two basic questions concerning these equations:

(A) When do solutions exist?

(B) What can one say about the set of all solutions?

The answers to both questions turn out to be interesting.

Based on the results for the Hermitian-Einstein equation one might expect to find a relation between solutions to our vortex equation and some type of stability for holomorphic bundles. In [ $\mathrm{Br} 1]$ and $[\mathrm{Br} 2]$ we studied question $(\mathrm{A})$ and gave the necessary and sufficient conditions for existence of solutions. We showed there that these can indeed be linked to a stability property of a holomorphic bundle with a prescribed holomorphic section. Given such a pair $(E, \phi)$, the appropriate notion of stability (which we call $\phi$-stability) can be expressed in terms of the slopes of subsheaves of $E$. The standard notion of stability involves a comparison of slopes of subsheaves with the slope of $E$. In the presence of a holomorphic section the correct notion of stability turns out to involve a comparison of slopes of subsheaves with slopes of quotients of $E$ by subsheaves which contain the section. The results of $[\mathrm{Br} 1]$ show that when the bundle $(E, \phi)$ is $\phi$-stable there is a range for the parameter $\tau$ within which the Yang-Mills-Higgs functional can attain its absolute minimum, i.e. within which the vortex equation has a solution. Conversely, when the vortex equation has a solution, the bundle must split as a direct sum of a $\phi$-stable component plus a sum of stable components.

In this paper we provide an answer to the second question in the case where the bundle is a line bundle. Our main result shows that the moduli space of global 
minima depends on the value of the parameter $\tau$ in the functional. There is a critical value determined by the first Chern class of the bundle and the volume of the base manifold. For $\tau$ below the critical value the moduli space is empty. For $\tau$ above the critical value it is in 1-1 correspondence with a certain subset of the set of effective divisors on the base manifold. When $\tau$ is at the critical value the moduli space is 1-1 correspondence with a certain subset of the set of linear equivalence classes of all divisors on the base. In the case where the base is a compact Riemann surface these results complement rather well the picture of classical vortices over $\mathbf{R}^{2}$.

\section{Definition of the Functional}

Let $X$ be a closed Kähler manifold of complex dimension $n$. Fix a Kähler metric on $X$ and let $\omega$ be the associated Kähler form. Let $E$ be a rank $R$ complex vector bundle over $X$. We will usually consider the vector bundle $E$ to be endowed with a fixed hermitian metric $H$. Let $\Omega^{0}(X, E)$ denote the smooth sections of $E$ and let $\mathscr{A}(H)$ denote connections on $E$ that are unitary with respect to $H$. We will also need to consider the spaces $\Omega^{p, q}(X, E)$ and $\Omega^{p, q}(X$, End $E)$, i.e. the spaces of forms of holomorphic type $(p, q)$ with values in $E$ and in the endomorphism bundle of $E$ respectively. These spaces all carry hermitian metrics induced by the Kähler metric on $X$ and the metric on $E$. Using the metric on $E$ we get identifications $E \approx E^{*}$ and also $E \otimes E^{*} \approx$ End $E$.

Definition 1.1. We define the Yang-Mills-Higgs functional

$$
\mathrm{YMH}_{\tau}: \mathscr{A}(H) \times \Omega^{0}(X, E) \rightarrow \mathbf{R}
$$

by

$$
\mathrm{YMH}_{\tau}(D, \phi)=\left\|F_{D}\right\|_{L^{2}}^{2}+\|D \phi\|_{L^{2}}^{2}+\frac{1}{4}\left\|\phi \otimes \phi^{*}-\tau \mathbf{I}\right\|_{L^{2}}^{2} .
$$

Here $F_{D} \in \Omega^{2}(X$, End $E)$ is the curvature of the connection $D, D \phi \in \Omega^{1}(X, E)$ is the covariant derivative, $I \in \Omega^{0}(X$, End $E) \approx \Omega^{0}\left(X, E \otimes E^{*}\right)$ is the identity section and $\tau$ is a real parameter. The adjoint of $\phi$ is taken with respect to $H$.

\section{Remarks.}

1. This functional should be compared to the classical Yang-Mills-Higgs functional over $d$-dimensional Euclidean space (cf. $[\mathrm{J}-\mathrm{T}]$ ). The major difference in the form of the functional is the presence of the real parameter $\tau$. We need to introduce this extra parameter because, unlike $\mathbf{R}^{d}$, our base space $X$ is compact. This is explained more fully later, in Sect. 5 . We shall see that varying $\tau$ corresponds to scaling the volume of $X$.

2. The functional $\mathrm{YMH}_{\tau}(D, \phi)$ is invariant under the standard action of the unitary gauge group $\mathscr{G}$ on $\mathscr{A}(H) \times \Omega^{0}(X, E)$. The functional can thus be thought of as being defined on $\mathscr{B}=\left(\mathscr{A}(H) \times \Omega^{0}(X, E)\right) / \mathscr{G}$.

\section{A Lower Bound on $\mathrm{YMH}_{\tau}$ and the Vortex Equation}

Using the Kähler structure on $X$ we can rewrite $\mathrm{YMH}_{\tau}$ in a form that gives explicit information about its global minima. Recall (cf. [W]) that on a Kähler manifold 
with Kähler form $\omega$ we can define a map

$$
L: \Omega^{p, q}(X, \mathbb{C}) \rightarrow \Omega^{p+1, q+1}(X, \mathbb{C})
$$

by

$$
L(\alpha)=\alpha \wedge \omega .
$$

The $L^{2}$-adjoint of this map is denoted by $\Lambda$. In general

$$
\Lambda: \Omega^{p, q}(X, \mathbb{C}) \rightarrow \Omega^{p-1, q-1}(X, \mathbb{C}) .
$$

On $(1,1)$ forms $\Lambda$ is given by

$$
\Lambda \alpha=(\alpha, \omega)_{\omega}
$$

Here $(,)_{\omega}$ denotes the pointwise inner product induced on $(1,1)$ forms by the Kähler metric on $X$. If $d=\partial+\bar{\partial}$ is the splitting of the exterior derivative according to holomorphic type, then the operators $\partial, \bar{\partial}$, their $L^{2}$ adjoints and $\Lambda$ are related by the Kähler identities. These extend to unitary connections on complex bundles over $X$ so that if $D=D^{0,1}+D^{1,0}$ is such a connection, then

$$
\begin{aligned}
\sqrt{-1}\left[\Lambda, D^{0,1}\right] & =\left(D^{1,0}\right)^{*}, \\
-\sqrt{-1}\left[\Lambda, D^{1,0}\right] & =\left(D^{0,1}\right)^{*} .
\end{aligned}
$$

The splitting of $D$ into $D^{0,1}+D^{1,0}$ corresponds to the splitting $\Omega^{1}(X, E)=$ $\Omega^{0,1}(X, E) \oplus \Omega^{1,0}(X, E)$ coming from the complex structure on $X$. The adjoints are now with respect to the $L^{2}$ inner product on $\Omega^{p, q}(X, E)$ induced by the bundle metric and the Kähler metric on $X$.

Proposition 2.1. The functional

$$
\mathrm{YMH}_{\tau}: \mathscr{A}(H) \times \Omega^{0}(X, E) \rightarrow \mathbf{R}
$$

can be written as

$$
\begin{aligned}
\mathrm{YMH}_{\tau}(D, \phi)= & 4\left\|F_{D}^{02}\right\|_{L^{2}}^{2}+2\left\|D^{0,1} \phi\right\|_{L^{2}}^{2}+\left\|\sqrt{-1} \Lambda F_{D}+\frac{1}{2} \phi \otimes \phi^{*}-\frac{\tau}{2} \mathbf{I}\right\|_{L^{2}}^{2} \\
& +\tau \int_{X} \sqrt{-1} \operatorname{Tr}\left(F_{D}\right) \wedge \omega^{[n-1]}+\int_{X} \operatorname{Tr}\left(F_{D} \wedge F_{D}\right) \wedge \omega^{[n-2]} .
\end{aligned}
$$

Here $\omega^{[m]}=\frac{\omega^{m}}{(m) !}$ and $F_{D}^{02}$ is the component of $F_{D}$ of type $(0,2)$.

Proof. This follows from the identities

$$
\left\langle\sqrt{-1} \Lambda F_{D}, \phi \otimes \phi^{*}\right\rangle=-\left\|D^{0,1} \phi\right\|_{L^{2}}^{2}+\left\|D^{1,0} \phi\right\|_{L^{2}}^{2},
$$

and

$$
\left|F_{D}\right|^{2} \omega^{[n]}=\left|\Lambda F_{D}\right|^{2} \omega^{[n]}+\operatorname{Tr} F_{D} \wedge F_{D} \wedge \omega^{[n-2]}+2\left(\left|F_{D}^{02}\right|^{2}+\left|F_{D}^{20}\right|^{2}\right) .
$$

To get the first identity write

$$
\begin{aligned}
\left\langle\sqrt{-1} \Lambda F_{D}, \phi \otimes \phi^{*}\right\rangle & =\left\langle\sqrt{-1} \Lambda F_{D} \phi, \phi\right\rangle \\
& =\left\langle\sqrt{-1} \Lambda D^{0,1} D^{1,0} \phi+\sqrt{-1} \Lambda D^{1,0} D^{0,1} \phi, \phi\right\rangle,
\end{aligned}
$$


and then apply the Kähler identities (2.1). The inner products here are the $L^{2}$ inner products induced on $\Omega^{p, q}(X$, End $E)$ and $\Omega^{p, q}(X, E)$ respectively by the bundle metric on $E$ and the Kähler metric on $X$. The second identity is well known and can be obtained by straightforward calculation in suitable local coordinates (cf. for example [U]). In the case where $D$ is a unitary connection we have $\left|F_{D}^{02}\right|^{2}=\left|F_{D}^{20}\right|^{2}$ so the last term in (2.4) is $4\left|F_{D}^{02}\right|^{2}$.

One can now expand

$$
\begin{aligned}
\left\|\sqrt{-1} \Lambda F_{D}+\frac{1}{2} \phi \otimes \phi^{*}-\frac{\tau}{2} \mathbf{I}\right\|_{L^{2}}^{2}= & \left\|\sqrt{-1} \Lambda F_{D}\right\|_{L^{2}}^{2}+\left\langle\sqrt{-1} \Lambda F_{D}, \phi \otimes \phi^{*}\right\rangle \\
& -\tau\left\langle\sqrt{-1} \Lambda F_{D}, \mathbf{I}\right\rangle+\frac{1}{4}\left\|\phi \otimes \phi^{*}-\tau, \mathbf{I}\right\|_{L^{2}}^{2},
\end{aligned}
$$

and use the identities (2.3) and (2.4) to show that the expression in (2.2) is indeed equivalent to the one in (1.1). Notice that by definition

$$
\left\langle\sqrt{-1} \Lambda F_{D}, \mathbf{I}\right\rangle=\frac{\sqrt{-1}}{2 \pi} \int_{X} \operatorname{Tr}\left(F_{D}, \omega\right)_{\omega} \omega^{[n]}=\int_{X} \sqrt{-1} \operatorname{Tr}\left(F_{D}\right) \wedge \omega^{[n-1]} .
$$

The last two terms in (2.2) do not depend on the connection $D$. In fact Chern-Weil formulae show that they are determined by the first Chern class and second Chern character of $E$ respectively:

Lemma 2.2. Let $c_{1}(E) \in H^{2}(X, \mathbf{R})$ and $c h_{2}(E) \in H^{4}(X, \mathbf{R})$ be the first Chern class and second Chern character of $E$ respectively. Then

$$
C_{1}(E, \omega) \equiv \int_{X} c_{1}(E) \wedge \omega^{[n-1]}=\frac{\sqrt{-1}}{2 \pi} \int_{X} \operatorname{Tr}\left(F_{D}\right) \wedge \omega^{[n-1]},
$$

and

$$
C h_{2}(E, \omega) \equiv \int_{X} c h_{2}(E) \wedge \omega^{[n-2]}=-\frac{1}{8 \pi^{2}} \int_{X} \operatorname{Tr}\left(F_{D} \wedge F_{D}\right) \wedge \omega^{[n-2]}
$$

Proof. See for example [G].

Note. $C_{1}(E, \omega)$ is also known as $\operatorname{deg}(E, \omega)$.

An immediate corollary is the following:

Corollary 2.3. The functional $\mathrm{YMH}_{\tau}$ is bounded below by

$$
2 \pi \tau C_{1}(E, \omega)-8 \pi^{2} C h_{2}(E, \omega) \text {. }
$$

This (topological) lower bound is attained at $(D, \phi) \in \mathscr{A}(H) \times \Omega^{0}(X, E)$ if and only if

$$
\begin{gathered}
F_{D}^{02}=0, \\
D^{0,1} \phi=0, \\
\sqrt{-1} \Lambda F_{D}+\frac{1}{2} \phi \otimes \phi^{*}=\frac{\tau}{2} \mathbf{I} .
\end{gathered}
$$

Remarks. The first equation is an integrability condition on $D^{0,1}$. By results of Newlander-Nirenberg on integrable almost complex structures, this condition 
ensures that $D^{0,1}$ determines a holomorphic structure on $E$ (cf. [Ko]). The second equation then says that $\phi$ is holomorphic with respect to this holomorphic structure. The third equation generalises the Hermitian-Yang-Mills equation (which is recovered by taking $\phi=0$ ) and is the analog of the classical Vortex Equation over $\mathbf{R}^{2}$. For these reasons we refer to (2.6c) as either the Hermitian-Yang-Mills-Higgs or the Vortex equation.

\section{Statement of Problem from Two Equivalent Points of View}

In order to find the absolute minima characterized by Eqs. (2.6a), (2.6b) and (2.6c), one may proceed as follows. Firstly, we consider only integrable unitary connections, i.e. those which belong to $\mathscr{A}^{1,1}(H)$, where

$$
\mathscr{A}^{1,1}(H)=\left\{D \in \mathscr{A}(H) / F_{D}^{02}=0\right\} .
$$

We then look for minimizing pairs $(D, \phi)$ in $\mathscr{S} \subset \mathscr{A}^{1,1}(H) \times \Omega^{0}(X, E)$, where

$$
\mathscr{S}=\left\{(D, \phi) \in \mathscr{A}^{1,1}(H) \times \Omega^{0}(X, E) / D^{0,1} \phi=0\right\} .
$$

The problem to be solved thus becomes:

Statement of Problem. Given a complex bundle $E$ with fixed Hermitian metric $H$, find all pairs $(D, \phi) \in \mathscr{S}$ such that the Vortex equation $(2.6 c)$ is satisfied for a given value of $\tau$.

These pairs, which are the minima of the functional $\mathrm{YMH}_{\tau}$ on $\mathscr{A}(H) \times \Omega^{0}(X, E)$, will be called $\tau$-Vortex pairs. We can define the set of all such pairs as follows:

Definition 3.1. Let $E$ be a complex line bundle over $X$. Let $H$ be a fixed Hermitian metric on $E$. Let $\mathrm{YMH}_{\tau}$ be given by (1.1). Define the space of $\tau$-Vortex pairs on $(E, H)$ by

$$
\mathscr{U}_{\tau}(H)=\left\{(D, \phi) \in \mathscr{A}(H) \times \Omega^{0}(X, L) / \mathrm{YMH}_{\tau}(D, \phi)=2 \pi \tau C_{1}(E, \omega)-8 \pi^{2} C h_{2}(E, \omega)\right\} .
$$

We have already noted that the Yang-Mills-Higgs functional is invariant under the action of the unitary gauge group $\mathscr{G}$. This means that $\mathscr{U}_{\tau}(H)$ is a $\mathscr{G}$-invariant set and that $\tau$-vortices can be defined by equivalence classes in $\mathscr{B}=(\mathscr{A}(H) \times \Omega(X, E)) / \mathscr{G}$.

Definition 3.2. We define the space of (unitary) gauge equivalence classes of $\tau$-Vortex pairs by

$$
\mathscr{V}_{\tau}(H)=\left\{[D, \phi] \in \mathscr{B} / \mathrm{YMH}_{\tau}(D, \phi)=2 \pi \tau C_{1}(E, \omega)-8 \pi^{2} C h_{2}(E, \omega)\right\} .
$$

The problem as stated above is thus to understand $\mathscr{V}_{\tau}(H)$. We do not however attempt to solve the problem in this form. Our strategy is to make use of some standard complex differential geometry in order to reformulate the problem in a more tractable from. The basic fact that we use is that given a holomorphic structure and a hermitian metric on a complex bundle, there is a unique complex connection compatible with both. This means, on the one hand, that given $D \in \mathscr{A}^{1,1}(H), D$ is the unique metric connection compatible with the metric $H$ and the holomorphic structure determined by $D^{0,1}$. On the other hand, it allows us to treat Eqs. (2.6a), (2.6b) and (2.6c) as conditions on 
(i) a holomorphic structure on $E$ given by a $\bar{\partial}$-operator

$$
\bar{\partial}_{E}: \Omega^{0}(X, E) \rightarrow \Omega^{0,1}(X, E),
$$

(ii) a hermitian bundle metric $H$,

(iii) a section $\phi \in \Omega^{\circ}(X, E)$.

In terms of the triple $\left(\bar{\partial}_{E}, \phi, H\right)$, the equations can be expressed as

$$
\begin{gathered}
\bar{\partial}_{E} \phi=0, \\
\sqrt{-1} \Lambda F_{\bar{\partial}, H}+\frac{1}{2} \phi \otimes \phi^{* H}-\frac{1}{2} \tau \mathbf{I}=0 .
\end{gathered}
$$

Here $F_{\bar{\partial}, H}$ is the curvature of the metric connection compatible with $\bar{\partial}_{E}$ and $H$. The notation $\phi^{* H}$ emphasizes that the adjoint is taken with respect to the metric $H$. Let

$$
T_{\tau}=\left\{\left(\bar{\partial}_{E}, \phi, H\right) / \text { Eqs. (3.5a) and (3.5b) are satisfied }\right\} .
$$

One way we can look for solutions to these equations is by picking a pair $\left(\bar{\partial}_{E}, \phi\right)$ which satisfies (3.5a) and then trying to solve (3.5b) for the metric $H$. We will call such metrics $\tau$-Hermitian-Yang-Mills-Higgs metrics. The problem to be solved then becomes:

Restatement of Problem. Given $\left(E, \bar{\partial}_{E}, \phi\right)$, i.e. a holomorphic bundle $E$ with a prescribed holomorphic section $\phi$, determine whether the bundle supports $\tau$-Hermitian-YangMills-Higgs metrics.

This is the approach taken in [Br1] and is the method we will use in Sect. 4 to study the set $T_{\tau}$ for a line bundle. What we have thus done is to transform the problem of minimizing the functional $\operatorname{YMH}_{\tau}(D, \phi)$ defined on $(E, H)$, a complex bundle with fixed metric, into the problem of finding a special metric on $\left(E, \bar{\partial}_{E}, \phi\right)$, i.e. on a holomorphic bundle with a prescribed holomorphic section.

In fact these two problems are equivalent. However before we can see the corespondence between them we must take into account an invariance in $T_{\tau}$ which becomes from the action of the complex gauge group $\mathscr{G}_{\mathbb{C}}$.

It is well known that any two metrics $H$ and $K$ are related by $K=H h$, where $h \in \Omega^{\circ}(X$, End $E)$ is positive and self adjoint with respect to $H$. Furthermore $h$ can be decomposed as $h=g^{*} g$, where $g \in \mathscr{G}_{\mathbb{C}}$. Up to unitary gauge transformations this splitting in unique (cf. [Ko], [D3]). The gauge transformation $g$ is nothing more than the change of basis from a unitary frame for $H$ to a unitary frame for $K$. Now consider the action of $\mathscr{G}_{\mathbb{C}}$ on $\Omega^{\circ}(X, E)$ and on $\mathscr{C}$, the space of integrable $\bar{\partial}$-operators on $E$. The complex gauge group acts on both these spaces by "pushforward," i.e.

$$
\begin{aligned}
& g(\bar{\partial})=g \circ \bar{\partial} \circ g^{-1}, \\
& g(\phi)=g \phi .
\end{aligned}
$$

Proposition 3.3. Let $\left(\bar{\partial}_{E}, \phi, H\right) \in T_{\tau}$. Then for every $g \in \mathscr{G}_{\mathbb{C}},\left(g^{-1}\left(\bar{\partial}_{E}\right), g^{-1} \phi, H g^{*} g\right)$ is also in $T_{\tau}$.

Before proving the proposition we first prove a lemma about the action of $\mathscr{G}_{\mathbb{C}}$ on $\mathscr{A}$, the space of connections. Of course $\mathscr{G}_{\mathbb{C}}$ has a simple pushforward action on $\mathscr{A}$. However it has a second action on $\mathscr{A}$ defined by

$$
g(D)=g^{\circ} D^{0,1} \circ g^{-1}+\left(g^{*}\right)^{-1} \circ D^{1,0} \circ g^{*} .
$$


This action has the advantage of being compatible with the construction of metric connections.

Lemma 3.4. Let $D_{\bar{\partial}_{E}, H}$ denote the metric connection compatible with $\bar{\partial}_{E}$ and $H$. Let $\mathscr{G}_{\mathbb{C}}$ act on $\mathscr{C}$ and $\mathscr{A}$ by (3.7) and (3.8) respectively. Let $g \in \mathscr{G}_{\mathbb{C}}$ and let $h=g^{*} g$. Then

(i) $D_{g\left(\bar{\partial}_{E}\right), H}=g\left(D_{\bar{\partial}_{E}, H}\right)$, i.e. the set of metric connections is $\mathscr{G}_{\mathbb{C}}$ invariant with respect to the action of $\mathscr{G}_{\mathbb{C}}$ given by (3.8),

(ii) $g\left(D_{\bar{\partial}_{E}, H}\right)=g \circ D_{\bar{\partial}_{E}, H h} \circ g^{-1}$,

(iii) $F_{g\left(\bar{\partial}_{E}\right), H}=g \circ F_{\bar{\partial}_{E}, H h} \circ g^{-1}$.

Proof. (i) Splitting $D_{\bar{\partial}_{E}, H}$ into its $(0,1)$ and $(1,0)$ parts we can write $D_{\bar{\partial}_{E}, H}=\bar{\partial}_{E}+D_{H}^{\prime}$, where by the Kähler identities $D_{H}^{\prime *}=\sqrt{-1}\left[\Lambda, \bar{\partial}_{E}\right]$. Hence

$$
g\left(D_{\bar{\partial}_{E}, H}\right)=g \circ \bar{\partial}_{E} \circ g^{-1}+\left(g^{*}\right)^{-1} \circ D_{H}^{\prime} \circ g^{*} \text {. }
$$

On the other hand

$$
D_{g\left(\bar{\partial}_{E}\right), H}=g\left(\bar{\partial}_{E}\right)+D_{H, g}^{\prime}
$$

where $\sqrt{-1}\left[\Lambda, g\left(\bar{\partial}_{E}\right)\right]=\left(D_{H, g}^{\prime}\right)^{*}$. Now $g\left(\bar{\partial}_{E}\right)=g \circ \bar{\partial}_{E}^{\circ} g^{-1}$ so to prove (i) we need only show that $\left(g^{*}\right)^{-1} \circ D_{H}^{\prime} \circ g^{*}=D_{H, g}^{\prime}$.

But

$$
\sqrt{-1}\left[\Lambda, g\left(\bar{\partial}_{E}\right)\right]=g \circ \sqrt{-1}\left[\Lambda, \bar{\partial}_{E}\right] \circ g^{-1},
$$

since $\Lambda$ and $g$ commute. By the Kähler identities this is a equivalent to what we need.

(ii) A calculation (using a local holomorphic frame for $E$ ) shows that

$$
D_{\bar{\partial}_{E}, H h}=D_{\bar{\partial}_{E}, H}+h^{-1} D_{H}^{\prime}(h)=\bar{\partial}_{E}+h^{-1} D_{H}^{\prime} h .
$$

From (3.9) we see that $g^{-1} \circ g\left(D_{\bar{\partial}_{E}, H}\right) \circ g=\bar{\partial}_{E}+\left(g^{*} g\right)^{-1} \circ D_{H}^{\prime} \circ g^{*} g$. Hence if $h=g^{*} g$ then (ii) follows.

(iii) For any $D \in \mathscr{A}^{1,1}$ with curvature $F(D)$ we have

$$
F\left(g \circ D \circ g^{-1}\right)=g \circ F(D) \circ g^{-1} \text {. }
$$

Thus

$$
F_{g\left(\bar{\partial}_{E}\right), H}=F\left(g \circ D_{\bar{\partial}_{E}, H h} \circ g^{-1}\right)=g \circ F_{\bar{\partial}_{E}, H h} \circ g^{-1} .
$$

Proof of Proposition 3.3. The proposition is equivalent to the statement that the triple $\left(\bar{\partial}_{E}, \phi, H h\right)$ satisfies $(3.5 \mathrm{a})$ and $(3.5 \mathrm{~b})$ if and only if $\left(g\left(\bar{\partial}_{E}\right), g \phi, H\right)$ does. Here $h=g^{*} g$. Now by Lemma 3.4,

$\sqrt{-1} \Lambda F_{g\left(\bar{\partial}_{E}\right), H}-\frac{1}{2}(g \phi) \otimes(g \phi)^{* H}+\frac{1}{2} \tau \mathbf{I}=g^{\circ}\left[\sqrt{-1} \Lambda F_{\bar{\partial}_{E}, H h}-\frac{1}{2} \phi \otimes \phi^{* H h}+\frac{1}{2} \tau \mathbf{I}\right] \circ g^{-1}$. Also, $g\left(\bar{\partial}_{E}\right)(g \phi)=g \bar{\partial}_{E}(\phi)$. This proves the proposition.

As an immediate corollary we obtain the correspondence between the two versions of our problem.

Corollary 3.5. Let $h=g^{*} g$, where $g$ is an element of the complex gauge group $\mathscr{G}_{\mathbb{C}}$. Then the following are equivalent:

(1) The triple $\left(\bar{\partial}_{E}, \phi, H h\right)$ satisfies Eqs (3.5a) and (3.5b).

(2) The pair $\left(D_{g\left(\bar{\partial}_{E}\right), H}, g \phi\right)$ is a $\tau$-Vortex Pair on $(E, H)$, i.e. gives a minimum for the functional $\mathrm{YMH}_{\tau}: \mathscr{A}(H) \times \Omega^{0}(X, E) \rightarrow \mathbf{R}$. 
Proof. By Proposition (3.3) $\left(\bar{\partial}_{E}, \phi, H h\right) \in T_{\tau}$ if and only if $\left(g\left(\bar{\partial}_{E}\right), g \phi, H\right) \in T_{\tau}$. But the latter condition is simply a restatement of (2).

We can rephrase this result in a somewhat more elegant fashion if we make the following definition.

Definition 3.6. Define an equivalence relation on $T_{\tau}$ by

$$
\left(\bar{\partial}_{E}, \phi, H\right) \sim\left(g^{-1}\left(\bar{\partial}_{E}\right), g^{-1} \phi, H g^{*} g\right)
$$

for any $g \in \mathscr{G}_{\mathbb{C}}$. Let $\mathscr{T}_{\tau}=T_{\tau} / \sim$ be the set of equivalence classes of solutions to (3.5a) and $(3.5 b)$.

Proposition 3.7. Let $E$ be a complex bundle over the Kähler manifold $X$. Fix a hermitian metric $H$ on $E$. Then there is a 1-1 correspondence between $\mathscr{V}_{\tau}(H)$ and $\mathscr{T}_{\tau}$.

Proof. The map from $\mathscr{V}_{\tau}(H) \mathscr{T}_{\tau}$ is given by

$$
[D, \phi] \rightarrow\left[D^{0,1}, \phi, H\right] \text {. }
$$

The inverse map from $\mathscr{T}_{\tau}$ to $\mathscr{V}_{\tau}(H)$ is defined as follows: Let $\left[\bar{\partial}_{E}, \phi, K\right]$ be an element in $\mathscr{T}_{\tau}$. Say $K=H g^{*} g$ for some $g \in \mathscr{G}_{\mathbb{C}}$. The $\left(\bar{\partial}_{E}, \phi, K\right) \sim\left(g\left(\bar{\partial}_{E}\right), g \phi, H\right)$ and we can map

$$
\left[\bar{\partial}_{E}, \phi, K\right] \rightarrow\left[D\left(g\left(\bar{\partial}_{E}\right), H\right), g \phi\right] .
$$

Using Lemma 3.4 it is straightforward to check that these maps are well defined and are indeed inverse of one another.

\section{Special Case of Rank $E=1$}

The problem of finding Hermitian-Yang-Mills-Higgs metrics on holomorphic bundles with prescribed holomorphic sections has been discussed in general in [Br1]. It was shown there that the existence of such metrics corresponded to a stability property for holomorphic bundles. In the special case of holomorphic line bundles we can be more specific and can give a full description on the moduli space of solutions to the Vortex (or Hermitian-Yang-Mills-Higgs) equation. In addition we can analyse the equation in a much more direct fashion than was necessary for the general case.

Let $L$ be a holomorphic line bundle over $X$, with the holomorphic structure being given by

$$
\bar{\partial}_{L}: \Omega^{0}(X, L) \rightarrow \Omega^{0,1}(X, L) .
$$

Let $\phi \in \Omega^{\circ}(X, L)$ be a holomorphic section of $L$. Let $H$ be a given background hermitian metric on $L$. We have already noted that any other hermitian metric on $L$ is related to $H$ by a positive, self-adjoint bundle endomorphism. Since $L$ is a line bundle, this means that the two metrics are related by a positive real valued function $h \in C^{\infty}(X, \mathbf{R})$. Given a metric $K$, we may thus write $K=H e^{2 u}$, where $u \in C^{\infty}(X, \mathbf{R})$. The factor 2 is purely for later convenience.

Lemma 4.1. If $K=H e^{2 u}$ then the vortex equation (3.5b), as an equation for $u$, is

$$
\Delta u+\frac{1}{2}|\phi|_{H}^{2} e^{2 u}+\left(\sqrt{-1} \Lambda F_{H}-\frac{1}{2} \tau\right)=0 .
$$


Here $\Delta$ is the (positive definite) Laplacian on $X$.

Proof. If $K=H h$ then a straightforward calculation shows that

$$
\sqrt{-1} \Lambda F_{K}=\sqrt{-1} \Lambda F_{H}+\sqrt{-1} \Lambda \bar{\partial}_{L}\left(h^{-1} D_{H}^{\prime}(h)\right)
$$

Here $F_{K}$ is the curvature of the connection determined by a fixed $\bar{\partial}$-operator $\bar{\partial}_{L}$ on $L$ and the metric $K$. Also, $D_{H}^{\prime}$ is the $(1,0)$ part of the connection induced by $D_{H}$ on End $L$. Since $L$ is a line bundle, End $L$ is a trivial bundle and $D_{H}=d$ on End $L$. Hence if $h=e^{2 u}$ then

$$
\sqrt{-1} \Lambda F_{K}=\sqrt{-1} \Lambda F_{H}+2 \sqrt{-1} \Lambda \bar{\partial} \partial(u)
$$

The Kähler identities give

$$
2 \sqrt{-1} \Lambda \bar{\partial} \partial(u)=2 \partial^{*} \partial(u) \equiv 2 \Delta^{\prime}(u)=\Delta(u) .
$$

Here $\Delta \equiv\left(d^{*} d+d d^{*}\right)$ which for functions on Euclidean $n$-space is $\Delta(f)=-\sum_{i=1}^{n} \frac{\partial^{2} f}{\partial x_{i}^{2}}$.

Finally, we note that using $H$ to identify $L^{*}$ with $L$, we can write $\phi \otimes \phi^{* H}=|\phi|_{H}^{2}$. Thus with $K=H e^{2 u}$,

$$
\phi \otimes \phi^{* K}=|\phi|_{K}^{2}=|\phi|_{H}^{2} e^{2 u} .
$$

Equation (4.1) is almost in the form of a non-linear PDE analysed by Kazdan and Warner [K-W] (WARNING: The Laplacian used by Kazdan and Warner is the negative definite operator, i.e. it differs from the one defined here by a minus sign). Let

$$
c=2 \int_{X}\left(\sqrt{-1} \Lambda F_{H}-\frac{1}{2} \tau\right)
$$

and choose $v \in C^{\infty}(X, \mathbf{R})$ to be a solution to

$$
-\Delta(v)=\left(\sqrt{-1} \Lambda F_{H}-\frac{1}{2} \tau\right)-\frac{1}{2} c .
$$

Define

$$
w=2(u-v) .
$$

Then $u$ is a solution to (5.3) iff $w$ is a solution to

$$
-\Delta(w)-\left(\frac{1}{2}|\phi|_{H}^{2} e^{2 v}\right) e^{w}-c=0 .
$$

This equation is precisely of the form considered in $[\mathrm{K}-\mathrm{W}]$. The results of Kazdan and Warner that we will need are collected together in the next theorem.

Theorem 4.2. [K-W]. Let $M$ be any compact Riemannian manifold. Consider the equation

$$
-\Delta(u)+h e^{u}-c=0,
$$

where $h \in C^{\infty}(M, \mathbf{R})$ is not identically zero and $c$ is a real constant. Then

(i) if $c=0$ a necessary condition for existence of $u \in C^{\infty}(M, \mathbf{R})$ satisfying $(4.9)$ is that $h$ changes sign on $M$.

(ii) if $c>0$ a necessary condition for existence of $u \in C^{\infty}(M, \mathbf{R})$ satisfying (4.9) is that $h$ is strictly positive somewhere on $M$.

(iii) if $c<0$ and $h \leqq 0$ then there is a unique $u \in C^{\infty}(M, \mathbf{R})$ which satisfies (4.9). 
Proof. cf. Theorem 5.3, 7.2, 10.5(a) and Remark 10.2 in [K-W].

Theorem 4.3. Let $L$ be a holomorphic line bundle over an n-dimensional compact Kähler manifold $X$. Let $\phi \in \Omega^{\circ}(X, L)$ be a prescribed (non-trivial) holomorphic section of $L$. Then there exists a hermitian metric $K$ on $L$ satisfying the vortex equation $(3.5 b)$ if and only if

$$
\frac{\tau \mathrm{Vol}(X)}{4 \pi}>\mu(L) .
$$

Here $\mu(L)=C_{1}(L, \omega)$, i.e. $\mu(L)$ is the slope of $L$ with respect to the Kähler form $\omega$ on $X$. Proof. Fix a background metric $H$ on $L$. Let $K=H e^{2 u}$ and define $c \in \mathbf{R}$ as in (4.5) and $v \in C^{\infty}(X, \mathbf{R})$ as in (4.6). The vortex equation then becomes

$$
-\Delta(w)+h e^{w}-c=0,
$$

with

$$
h=-\left(\frac{1}{2} \mid \phi_{H}^{2} e^{2 v}\right) .
$$

By Theorem 4.2 this has a (unique) solution if and only if $c<0$, i.e.

$$
\int_{X}\left(\sqrt{-1} \Lambda F_{H}-\frac{1}{2} \tau\right)<0
$$

The result now follows from the Chern-Weil formula for $C_{1}(L, \omega)$ (cf. Lemma 2.2).

We can use Theorem 4.3 to get a complete description of the moduli space of all vortices.

Definition 4.4. Let $L$ be a complex line bundle over $X$ with $C_{1}(E, \omega)=N$ and with a fixed Hermitian metric $H$. Let the functional $\mathrm{YMH}_{\tau}$ be as in Definition 1.1. Define

$$
\mathscr{V}_{\tau}^{N}(H)=\left\{[D, \phi] \in \mathscr{B} / \mathrm{YMH}_{\tau}(D, \phi)=2 \pi N \tau\right\} .
$$

The set $\mathscr{V}_{\tau}^{N}$ is the set of $\tau$-Vortex Pairs on the Hermitian Line Bundle $(L, H)$.

The first thing to observe is that each vortex pair $[D, \phi]$ in $\mathscr{V}_{\tau}^{N}(H)$ determines an effective divisor of $X$, namely the zero set of the (holomorphic) section $\phi$. Conversely, an effective divisor determines a holomorphic line bundle together with a holomorphic section (cf. $[\mathrm{G}-\mathrm{H}]$ ). If $\mathscr{D}$ is the effective divisor, $L_{\mathscr{D}}$ the line bundle, and $\phi_{\mathscr{D}}$ the holomorphic section, then the zero set of $\phi_{\mathscr{D}}$ is precisely $\mathscr{D}$. Furthermore, $\mathscr{D}$ determines an element of $H_{2 n-2}(X, \mathbb{C})$ and thus by Poincare duality an element $\eta_{\mathscr{D}} \in H^{2}(X, \mathbb{C})$. The first Chern class $c_{1}\left(L_{\mathscr{D}}\right)$ is represented by $\eta_{\mathscr{D}}$. It follows that

$$
C_{1}\left(L_{\mathscr{D}}, \omega\right)=\int_{X} c_{1}\left(L_{\mathscr{D}}\right) \wedge \omega^{[n-1]}=\#\left(\mathscr{D},\left[\omega^{[n-1]}\right]\right),
$$

where $\left[\omega^{[n-1]}\right]$ is the 2-cycle dual to $\omega^{[n-1]}$ and \#(, ) denotes the intersection pairing. Definition 4.5. Let

$$
\operatorname{Div}(X)_{+}^{N}=\left\{\text { effective divisors on }(X, \omega) \text { with } \#\left(\mathscr{D},\left[\omega^{[n-1]}\right]\right)=N\right\} .
$$

It follows from the above discussion that given $\mathscr{D}$ in $\operatorname{Div}(X)_{+}^{N}$, one obtains a line bundle topologically equivalent to $L$, together with a holomorphic structure and a holomorphic section. We denote these by $\left(L_{\mathscr{D}}, \bar{\partial}_{\mathscr{D}}, \phi_{\mathscr{D}}\right)$. 
By Theorem 4.3, when $\tau>\frac{4 \pi N}{\operatorname{Vol}(X)}$, we can solve the vortex equation (3.5b) to obtain a metric $H_{\mathscr{D}}$ on $L_{\mathscr{D}}$ such that $\left(L_{\mathscr{D}}, \bar{\partial}_{\mathscr{D}}, \phi_{\mathscr{D}}\right)$ satisfy the vortex equation. What we want though is a vortex pair on our original bundle $(L, H)$. To obtain this we must make use of the (smooth) bundle isomorphism which relates $L_{\mathscr{D}}$ and $L$. This allows us to pull back the metric $H$ to a metric $H^{\prime}$ on $L_{\mathscr{D}}$, thereby turning the bundle equivalence into an isometry between $\left(L_{\mathscr{O}}, H^{\prime}\right)$ and $(L, H)$. The transformation between the two bundles is thus via an element of the unitary gauge group $\mathscr{G}$. Furthermore, if $H_{\mathscr{D}}$ and $H^{\prime}$ are related by the element $g \in \mathscr{G}_{\mathbb{C}}$ then (cf. Proposition 3.3) we can conclude that $\left(D_{g\left(\overline{(}_{\mathfrak{g})}\right), H^{\prime}}, g \phi_{\mathscr{D}}\right)$ is a $\tau$-Vortex Pair on $\left(L_{\mathscr{O}}, H^{\prime}\right)$. That is, it represents a (unitary) gauge equivalence class of vortex pairs on $(L, H)$. We have thus proven:

Theorem 4.6. For every $\tau>\frac{4 \pi N}{\operatorname{Vol}(X)}$ there is a 1-1 corresponding between $\mathscr{V}_{\tau}^{N}$ and $\operatorname{Div}(X)_{+}^{N}$. When $\tau$ is less than $\frac{4 \pi N}{\operatorname{Vol}(X)}, \mathscr{V}_{\tau}^{N}$ is empty.

The only remaining case to consider is when $\tau$ is equal to $\frac{4 \pi N}{\operatorname{Vol}(X)}$. In this case, integrating the vortex equation and using the Chern-Weil formula for $C_{1}(L, \omega)$ yields

$$
2 \pi N+\frac{1}{2} \int_{X}|\phi|_{H}^{2}=2 \pi N
$$

Hence the only solution possible has $\phi=0$ and $D \in \mathscr{A}^{1,1}(H)$ a solution to the Hermitian-Yang-Mills equation.

$$
\sqrt{-1} \Lambda F_{D}=\frac{2 \pi N}{\operatorname{Vol}(X)}
$$

If we fix a $\bar{\partial}$-operator on $L$ and treat (4.15) as an equation for the metric $H^{\prime}=H e^{u}$, we get

$$
\Delta u+\left(\sqrt{-1} \Lambda F_{H}-\frac{2 \pi N}{\operatorname{Vol}(X)}\right)=0 .
$$

This elliptic equation has a solution which is unique up to an arbitrary constant. Furthermore, if $u$ satisfies (4.16) and $e^{u}=g^{*} g$, then $D(g(\bar{\partial}), H)$ satisfies (4.15). Notice that scaling $H^{\prime}$ by an arbitrary constant has no effect on $D(g(\bar{\partial}), H)$. Hence for each choice of $\bar{\partial}$-operator on $L$ we get a unique solution in $\mathscr{A}^{1,1}(H)$. Changing the $\bar{\partial}$-operator by the action of an element of $\mathscr{G}_{\mathbb{C}}$ changes the corresponding Hermitian-Yang-Mills connection by at most a unitary gauge transformation. (This follows from Proposition 3.7 with $\phi \equiv 0$ and $\tau=\frac{4 \pi N}{\operatorname{Vol}(X)}$.) We thus have a correspondence between solutions to (4.15) and $\mathscr{G}_{\mathbb{C}}$ equivalence classes of $\bar{\partial}$-operators on $L$. But these are precisely the equivalence classes of holomorphic line bundles with $C_{1}(L, \omega)=N$. We see therefore that when $\tau$ is equal to $\frac{4 \pi N}{\operatorname{Vol}(X)}$ there is a 
1-1correspondence between $\mathscr{V}_{\tau}^{N}(H)$ and the holomorphic line bundles of degree $N$ over $X$. In the special case where $X$ is algebraic, all holomorphic line bundles are of the form $L_{\mathscr{D}}$ for some divisor $\mathscr{D}$ (cf. $[\mathrm{G}-\mathrm{H}]$ ). Also, two linearly equivalent divisors yield the same holomorphic line bundle. We have thus proved:

Theorem 4.7. If $\tau=\frac{4 \pi N}{\operatorname{Vol}(X)}$ then $\mathscr{V}_{\tau}^{N}(H)$ is in 1-1 correspondence with the holomorphic lines bundles over $X$ satisfying $C_{1}(L, \omega)=N$. If $X$ is algebraic and $\operatorname{Div}(X)^{N}$ denotes the set of all divisors on $(X, \omega)$ with \#(D, $\left.\left[\omega^{[n=1]}\right]\right)=N$, then there is a 1-1 correspondence between $\mathscr{V}_{\tau}^{N}(H)$ and the linear equivalence classes in $\operatorname{Div}(X)^{N}$.

Remark. If $X$ is a Riemann surface, then an element of $\operatorname{Div}(X)_{+}^{N}$ is given by a collection of $N$ (not necessarily distinct) points on $X$. The ordering of the collection is not significant. Hence in this case when $\tau>\frac{4 \pi N}{\operatorname{Vol}(X)}, \mathscr{V}_{\tau}^{N}(H)$ is in 1-1 correspondence with the $N$-fold symmetric product $\operatorname{Sym}^{N}(X)$. This can be compared to the case of classical abelian vortices over $\mathbf{R}^{2}$. In that case the space of vortices with vortex number $N$ is in 1-1 correspondence with $\operatorname{Sym}^{N}\left(\mathbf{R}^{2}\right)$.

\section{The Parameter $\tau$}

The results of the previous section show how the behaviour of the functional changes depending on the value of the parameter $\tau$. This parameter is, as we remarked earlier, a feature which has no counterpart in the theory of classical Abelian vortices over $\mathbf{R}^{2}$. It is natural to try to understand how the parameter enters into the description of vortices over closed Kähler manifolds.

One can clearly define the Yang-Mills-Higgs functional with $\tau$ fixed at 1 . Proposition 2.1 and Corollary 2.3 still hold with $\tau=1$ and in that case the vortex equation is

$$
\sqrt{-1} \Lambda F_{D}+\frac{1}{2} \phi \otimes \phi^{*}-\frac{1}{2} \mathbf{I}=0 .
$$

There is however an obvious obstruction to solving this equation. This can be seen by integrating the trace of the equation. $X$ is compact so the first term is $2 \pi C_{1}(E, \omega)$ and the last term is $\frac{\operatorname{Vol}(X)}{2}$. One gets

$$
2 \pi C_{1}(E, \omega)+\frac{1}{2}\|\phi\|_{L^{2}}^{2}=\frac{1}{2} R \operatorname{Vol}(X) .
$$

Thus solutions cannot exist unless

$$
\operatorname{Vol}(X)>\frac{4 \pi C_{1}(E, \omega)}{R}
$$

This obstruction can be removed by rescaling the metric on $X$. Suppose we rescale the metric by a constant factor so that the new Kähler form is

$$
\omega_{t}=t^{2} \omega
$$

The Hodge inner product on $\Omega^{p, q}(X, \mathbb{C})$ gets rescaled by a factor $t^{-2(p+q)}$. Hence 
if $\operatorname{Vol}_{t}(X)$ and $C_{1}\left(E, \omega_{t}\right)$ are calculated with the rescaled metric then

$$
\begin{gathered}
\operatorname{Vol}_{t}(X)=t^{2 n} \operatorname{Vol}(X), \\
C_{1}\left(E, \omega_{t}\right)=t^{2 n-2} C_{1}(E, \omega) .
\end{gathered}
$$

Hence, with large enough $t^{2}$, the condition

$$
\operatorname{Vol}_{t}(X)>\frac{4 \pi C_{1}\left(E, \omega_{t}\right)}{R}
$$

can be satisfied. With the parameter $\tau$ in the functional, the corresponding condition to be satisfied is

$$
\tau \operatorname{Vol}(X)>\frac{4 \pi C_{1}(E, \omega)}{R} .
$$

This condition is precisely the condition in Theorem 4.3 governing the existence of $\tau$-Hermitian-Yang-Mills-Higgs metrics on line bundles. The point we wish to make is that the necessity of this condition can be attributed to the compactness of $X$. Furthermore this condition can be met either by rescaling the Kähler metric or by adjusting the parameter $\tau$. Since we wish to keep the Kähler metric fixed we are forced to include the parameter $\tau$ in our functional. The next proposition shows that there is in fact an exact correspondence between these two options.

Proposition 5.1. (i) Let $E$ and $X$ be as before. For given Kähler form $\omega$ and real number $\tau$ let $\mathrm{YMH}(D, \phi ; \tau, \omega)$ denote the functional defined in Sect. 1 . Suppose the Kähler metric is rescaled by a constant factor so that the new Kähler form is $\omega_{t}=t^{2} \omega$. Then

$$
\mathrm{YMH}(D, \phi ; \tau, \omega)=t^{4-2 n} \mathrm{YMH}\left(D, \frac{\phi}{t} ; \frac{\tau}{t^{2}}, \omega_{t}\right)
$$
(ii) Furthermore $\{D, \phi, \tau, \omega\}$ satisfies the vortex equation iff $\left\{D, \frac{\phi}{t}, \frac{\tau}{t^{2}}, \omega_{t}\right\}$
does, i.e.

$$
\sqrt{-1} \Lambda F_{D}+\frac{1}{2} \phi \otimes \phi^{*}-\frac{1}{2} \tau \mathbf{I}=0
$$

if and only if

$$
\sqrt{-1} \Lambda_{t} F_{D}+\frac{1}{2} \frac{\phi}{t} \otimes \frac{\phi^{*}}{t}-\frac{1}{2} \frac{\tau}{t^{2}} \mathbf{I}=0
$$

Proof. (i) This is an immediate consequence of the way that the Hodge metric on $(p, q)$ forms rescales under rescaling of the Kähler metric.

(ii) All we have to show is that

$$
\Lambda_{t}=\frac{1}{t^{2}} \Lambda,
$$

where $\Lambda_{t}$ is the adjoint of wedging with $\omega_{t}$. Let $\alpha \in \Omega^{p, q}(X, \mathbb{C})$ and $\beta \in \Omega^{p-1, q-1}(X, \mathbb{C})$. Let $(,)_{t}$ denote the Hodge metric with respect to the metric corresponding to $\omega_{t}$. 
Then

$$
\begin{aligned}
\left(\Lambda_{t} \alpha, \beta\right)_{t} & =\left(\alpha, \beta \otimes \omega_{t}\right)_{t} \\
& =t^{2}(\alpha, \beta \wedge \omega)_{t} \\
& =t^{2-2(p+q)}(\alpha, \beta \wedge \omega)_{1} \\
& =t^{2-2(p+q)}(\Lambda \alpha, \beta)_{1} \\
& =t^{2-2(p+q)+2(p+q-2)}(\Lambda \alpha, \beta)_{t} \\
& =t^{-2}(\Lambda \alpha, \beta)_{t} .
\end{aligned}
$$

If we choose $t$ such that $t^{2}=\tau$ we get the required correspondence between $\mathrm{YMH}(\mathrm{D}, \phi ; \tau, \omega)$ and $\mathrm{YMH}\left(D, \frac{\phi}{t} ; 1, \omega_{t}\right)$.

We end by noting that $\tau$ gives an upper bound on $|\phi|^{2}$ for those $\phi \in \Omega^{0}(X, E)$ which belong to $\tau$-vortex pairs:

Proposition 5.2. Let $(D, \phi)$ be a $\tau$-vortex pair on the complex bundle $E$ with Hermitian metric $H$. Then.

$$
|\phi|_{H}^{2} \leqq \tau
$$

Proof. Since $D$ is unitary with respect to $H$ and $D^{0,1} \phi=0$, we have

$$
\bar{\partial} \partial|\phi|_{H}^{2}=\left(D^{0,1} D^{1,0} \phi, \phi\right)_{H}-\left(D^{1,0} \phi, D^{1,0} \phi\right)_{H} .
$$

But $D$ is also integrable, so $F_{D}=D^{0,1} D^{1,0}+D^{1,0} D^{0,1}$. It follows (again using the holomorphicity of $\phi$ ) that

$$
\bar{\partial} \partial|\phi|_{H}^{2}=\left(F_{D} \phi, \phi\right)_{H}-\left(D^{1,0} \phi, D^{1,0} \phi\right)_{H}
$$

We now use the Kähler identities on $X$ and get

$$
\Delta|\phi|_{H}^{2}=2 \sqrt{-1} \Lambda \bar{\partial} \partial|\phi|_{H}^{2}=\left(2 \sqrt{-1} \Lambda F_{D} \phi, \phi\right)_{H}-2 \sqrt{-1} \Lambda\left(D^{1,0} \phi, D^{1,0} \phi\right)_{H} .
$$

Here $\Delta$ is the positive definite Laplacian. Hence, if the vortex equation is satisfied, then

$$
\Delta|\phi|_{H}^{2}=\left(\tau-|\phi|_{H}^{2}\right)|\phi|_{H}^{2}-2 \sqrt{-1} \Lambda\left(D^{1,0} \phi, D^{1,0} \phi\right)_{H}
$$

This can be written as

$$
\left(-\Delta-|\phi|_{H}^{2}\right)\left(\tau-|\phi|_{H}^{2}\right)=-2 \sqrt{-1} \Lambda\left(D^{1,0} \phi, D^{1,0} \phi\right)_{H} .
$$

The right-hand side of (5.13) is less than or equal to zero (as a calculation in local coordinates will show). We can thus apply the maximum principle to the elliptic operator $\left(-\Delta-|\phi|_{H}^{2}\right)$. This yields the required result, namely

$$
\left(\tau-|\phi|_{H}^{2}\right) \geqq 0 \text {. }
$$

Acknowledgement. I wish to thank my thesis advisor Karen Uhlenbeck for introducing me to Gauge Theory and for the many occasions during the course of this work on which I have profited from her insights. 


\section{References}

[A-H-D-M] Atiyah, M., Hitchin, N., Drinfeld, V., Manin, Y.: Phys. Lett. 65A, 185-187 (1978)

[A-H-S] Atiyah, M., Hitchin, N., Singer, I.: Self duality in four dimensional Riemannian geometry. Proc. R. Soc. Lond. A362, 425-61, (1978)

[B1] Bleecker, D.: Gauge theory and variational principles. Reading, MA: AddisonWesley 1981

[Br1] Bradlow, S.: Special metrics and stability for holomorphic bundles with global sections. J. Diff. Geom (to appear)

[Br2] Bradlow, S.: Vortices on Kahler manifolds. Thesis, University of Chicago, 1988

[D1] Donaldson, S. K.: An application of gauge theory to the topology of 4-manifolds. J. Diff. Geom. 18, 269-316 (1983).

[D2] Donaldson, K. K.: Nahm's Equation and the classification of monopoles. Commun. Math. Phys. 96, 387-408 (1984)

[D3] Donaldson, S. K.: Anti-self-dual Yang-Mills connections over complex algebraic surfaces and stable bundles. Proc. Lond. Math. Soc. 50, 1-26 Berlin, Heidelberg, New York: 1985

[F-U] Freed, D., Uhlenbeck, K.: Instantons and Four-Manifolds. Berlin, Heidelberg, New York: Springer 1984

[G] Gilkey, P. B.: Invariance theory, the heat equation, and the Atiyah-Singer index theorem. Wilmington, Delaware: Publish or Perish 1984

[G-H] Griffiths, P., Harris, J.: Principles of algebra geometry. New York: John Wiley 1978

[G-L] Ginsburg, V. L., Landau, L. D.: Zh. Eksp. Theor. Fiz. 20, 1064 (1950)

[Hi] Hitchin, N.: On the construction of monopoles. Commun. Math. Phys. 89, 277-292 (1980)

[H-M] Hurtubise, J., Murray, M.: On the construction of monopoles for the classical groups. Commun. Math. Phys. 122, 35-89 (1989)

[J-T] Jaffe, A., Taubes, C.: Vortices and monopoles. Boston, Birkhäuser 1980

[K-W] Kazdan, J. L., Warner, F. W.: Curvature functions for compact 2-manifolds. Ann. Math. 2, 99, 14-47 (1978)

[Ko] Kobayashi, S.: Differential geometry of complex vector bundles. Princeton, New Jersey: Princeton University Press 1987

[P] Palais, R.: The geometrization of physics. Lecture Notes in Math. Institute of Math. National Tsing Hua Univ. Taiwan

[T] Taubes, C.: Arbitrary $N$-Vortex Solutions to the first order Ginsburg-Landau Equations. Commun. Math. Phys. 72, 277-292 (1980)

[U] Uhlenbeck, K.: A priori estimates for Yang-Mills fields. Preprint

[U-Y] Uhlenbeck, K., Yau, S.-T.: On the existence of Hermitian-Yang-Mills connections in stable vector bundles. Commun. Pure and Appl. Math. 39, 257-293 (1986)

[W] Wells, R. O.: Differential analysis on complex manifolds. Berlin, Heidelberg, New York: Springer 1980 
